\title{
Role Of Audit In Obstetrics And Other Specialties
}

Audit is defined as systematic critical analysis of the quality of care with comparison to stated, pre existing, agreed upon standards / norms / protocols, with the information then used to improve practice. The audit process goes through four important steps, analyzing practice (what are we doing?); setting up standards (what should we be doing?), comparing these two, implementing change, analyzing the outcome of change (reassessing practice). The loop is closed, completing the cycle, and the process is continued until the practice is as close to the standard as possible.

Audit has been an accepted and an important tool specially in obstetrics and paediatrics for the last several years. ${ }^{1,2}$ Audit is extremely important in these branches as any deficiency or deviation from the accepted standards of care may result in the loss of lives or chronic ill health for healthy women and/or their newborns. Audit differs from the other currently used methods in obstetrics such as case reviews, verbal autopsy, or confidential inquiries, to analyze maternal and/or perinatal deaths, morbidity and "near misses", while it can be an important tool to analyze these events. Although audit and research both require data collection, they essentially differ from each other; audit goes through a cycle comprising the above steps.

In today's obstetric practice, audit is gaining increasing importance for improving the quality of care, both in the developed and the developing countries. Now-a -days, it is common for every institution, at all levels, to have the clinical protocols, but compliance to these protocols is not observed uniformly in most institutions. The Reproductive Health Clinical Protocols developed by Family Health Division, His Majesty's Government of Nepal is available for all levels of the health care system, and forms an excellent resource material also for those in private practice. Access to the Internet and the electronic media including databases like the Medline and Popline have brought the obstetrician closer to the challenges of modern obstetric practice. As a guide to the practicing obstetrician for setting up standards, the Cochrane DataBase provides metaanalysis of various randomised-controlled trials of drugs and procedures used in obstetric practice. Publication based on these meta analyses is available ${ }^{3}$ and provides useful information in places where computer facility does not exist. These help the clinicians to set standards when they do not exist; however, it is preferable to set standards suitable to the local context, taking into consideration the available resources ${ }^{4}$. Audit can be done in every health facility at any level. Some institutions periodically carry out audit specially when a change is introduced in the usual practice, while in most others, it is triggered by events like maternal / neonatal death, stillbirth, high caesarean section rates, high rates of sepsis or eclampsia or any unusual event.

In the lower levels of the health care system, the process of audit, by ensuring compliance with the standard protocols ensures positive outcomes of the basic health care like prevention and treatment of anaemia, control of fits, prevention of post partum haemorrhage and sepsis. In the district level, audit of the emergency obstetric care services, and assessment of the maternal/ fetal indicators, will help to identify 
deficiencies and provide viable and sound recommendations for strengthening such services. In the higher level of health care system, audit will not only improve the quality of care, but percolate this effect down to the base of the health care system due to its immense potential for teaching.

Audit offers many advantages. The principles of audit, if applied correctly, help to improve clinical care as well as resource allocation, budgetary and long term planning, measurement of productivity and other management issues. It can be used to assess the performance of each obstetric unit, compare between different units and as examples while setting standards or to motivate change in practice. As audit is usually initiated locally, it offers objective criteria, provides local ownership, promotes local action and is less expensive. Audit is usually non-punitive and can be used as an excellent educational tool. It can reveal deficiencies in record keeping. However, audit results cannot be generalised, and is usually not representative of other health facilities. It requires standards and intervention hence, is not suitable for primary prevention.

In the present climate of public awareness and medical litigation, audit helps in the practice of evidence based medicine with sound knowledge, to provide informed choices to the patient ${ }^{5}$. This will continue to strengthen the faith and respect of the people for the doctors. The principles of audit, as used in obstetrics and paediatrics, if adopted by other specialties of the medical science, has a great potential to offer benefits to the patients and their doctors.

\section{Dr. Sudha Sharma, MBBS, DGO, MRCOG \\ Chief Editor, JNMA \\ Senior Consultant, Maternity Hospital \\ Preceptor, PGMECC, Kathmandu}

\footnotetext{
Maresh M. M, Khaled K. M, Audit in Obstetrics, Current Opin Obstet Gynecol, 8 (4):296-9

3 Enkin M, Keirse M.J.C, Chalmers I.,A Guide to Effective Care in Pregnancy and Childbirth Oxford University Press, first published 1989.

4 Graham W., Wagaaraachchi P, Penny G, Mc Caw- Binns A; et Al, Criteria for Clinical Audit of the Quality of Hospital- Based Obstetric Care in Developing Countries: Bull World Health Organ 2000; 78 (5): 614-20.

5 Advani P, Doctor Patient and the Law; Publisher: Books For All, New Delhi, India, 1998.
} 